\title{
Non-invasive Neuromodulation in Problem Gambling: What Are the Odds?
}

\author{
Anna E. Goudriaan ${ }^{1,2} \cdot$ Renée S. Schluter ${ }^{1}$
}

Published online: 20 July 2019

(C) The Author(s) 2019

\begin{abstract}
Purpose of Review Non-invasive neuromodulation as a potential therapeutic target in addiction treatment is a fast-growing, but nascent research field. With gambling disorder as the first behavioral addiction, the goal of this review is to provide an overview of the current state-of-the-art of neuromodulation in substance use disorders and gambling disorder.

Recent Findings Only a few neuromodulation studies in gambling disorder are present, most of these are single-session studies. Effects of rTMS on craving have been described, but large placebo effects are also present, indicating a need for larger, blinded, multiple-session neuromodulation trials.

Summary The field of neuromodulation in gambling is in its infancy, given the limited number of studies, with small sample sizes. The effects that neuromodulation can have on diminishing craving and improving cognitive functions in substance use disorders are promising. As these factors also play a role in relapse in gambling disorder, these findings in SUDs indicate that investment in larger studies in gambling disorder, focusing on both clinically relevant outcome measures and on intermediate working mechanisms like craving and cognitive functions, is warranted.
\end{abstract}

Keywords Non-invasive neuromodulation $\cdot$ Neurostimulation $\cdot$ Pathological gambling $\cdot$ Cognition $\cdot$ Craving $\cdot$ rTMS $\cdot$ tDCS

\section{Introduction}

Neuromodulation as an add-on treatment in addictive disorders is gaining momentum in clinical addiction research. In recent years, more and more small-scale trials, including clinical trials with surrogate clinical outcome measures like craving, and trials without an adequate control condition, have been performed. On the other hand, the number of large-scale sham-controlled trials with outcome measures focused on treatment success (e.g., reduced substance use;

This article is part of the Topical Collection on Gambling

Anna E. Goudriaan

a.e.goudriaan@amc.uva.nl; agoudriaan@gmail.com

Renée S. Schluter

r.s.schluter@amc.uva.nl

1 Amsterdam UMC, University of Amsterdam, Department of Psychiatry, Amsterdam Institute for Addiction Research, Amsterdam, The Netherlands

2 Arkin and Jellinek, Mental Health Care, Amsterdam, The Netherlands relapse) still is limited. With the introduction of gambling disorder into the category of substance-related and addictive disorders in the DSM-5, the question arises what these findings on neuromodulation in substance-related disorders imply for gambling disorder. In gambling disorder, studies of neurocognitive functioning have revealed similar impairments as in substance-related disorders $[1 \bullet, 2-7]$, most consistently in the areas of executive functioning like behavioral impulsivity-response inhibition, planning, cognitive flexibility, and more motivational cognitive functions related to reward processing or reward sensitivity, like decision-making and delay discounting. These cognitive functions are malleable by neuromodulation techniques $[8 \cdot \bullet]$, leading to the question whether neuromodulation may also be a technique which could improve neurocognitive functions in gambling disorders, and in this way, diminish relapse-by influencing some of the working mechanisms that lead to relapse [9-11]. This short review focuses on how neuromodulation studies in gambling disorders can benefit from the current state-of-theart in neuromodulation in substance-related disorders, and what areas are most promising for future studies employing neuromodulation in gambling disorder. 


\section{Non-invasive Neuromodulation in Addiction: Methods}

Two main types of non-invasive neuromodulation are currently employed in addictive disorders: transcranial magnetic stimulation (TMS) and transcranial direct current stimulation (tDCS). For these two types of neuromodulation, a variety of stimulation settings can be used, and different neural targets can be addressed. For TMS, repeated TMS (rTMS) induces brief electrical currents in the cortical tissue, due to brief magnetic pulses generated by the TMS coil. TMS can be applied at high frequency (usually $10-20 \mathrm{~Hz}$ ), leading to an increase in excitability of the targeted cortical area, or at low frequency (e.g., $1 \mathrm{~Hz}$ ), leading to a decrease in excitability of the targeted area in the brain [12]. High-frequency rTMS is an FDAapproved treatment method for treatment-resistant depression [13], and recently has also been approved by the FDA for obsessive compulsive disorder, as indicated on their website [14]. Besides high- and low-frequency stimulation, theta burst stimulation (TBS) is used as a distinct TMS protocol, in which patterned stimulation is applied, by bursts of very short trains of stimulation - with three pulses delivered with a frequency of $50 \mathrm{~Hz}$ and an inter-burst interval of $200 \mathrm{~ms}$ [15]. When applied as an intermittent protocol (iTBS), the effect is excitatory while with continuous TBS (cTBS) is inhibitory [15]. In rTMS studies in substance use disorders (SUD), the most frequent placement of the TMS coil is over the dorsolateral prefrontal cortex (DLPFC), either applied at the left or right side of the skull-although bilateral stimulation and other cortical sites are possible as well.

tDCS is a neuromodulation technique in which a small electrical current (1-2 mA) is induced through placement of a pair of saline-soaked sponge electrodes, which are again usually placed over the DLPFC. Unlike rTMS, which leads to action potentials in neuronal axons, tDCS only leads to modulation of neuronal excitability by this weak electrical current, through depolarization or hyperpolarization of the resting membrane potential [16]. In-depth review of all possible settings of rTMS and tDCS is beyond the scope of this article. For gambling disorder, a relevant question is whether neuromodulation techniques can add to the current arsenal in treatment methods. For this purpose, disease markers that have been related to the course of SUDs, or gambling disorder, and that have been studied in neuromodulation studies in SUDs, are summarized and discussed below. The current neuromodulation literature in SUDs can be divided into studies that have focused on the effects of neuromodulation on (1) craving, (2) cognitive functions, including executive functions and reward processing, and (3) treatment success related factors like relapse or treatment retention [for reviews, see $8 \bullet \bullet$, 17, 18]. Neuromodulation studies in gambling disorder are discussed where such studies exist, or else implications of findings in SUDs for GD are presented.

\section{Effects of Neuromodulation on Craving}

Studies using non-invasive neuromodulation in SUDs mainly focus on craving as an outcome measure. An extensive body of literature indicates that both TMS and tDCS reduce craving, as indicated in numerous literature reviews [18-25, 26•]. However, caution is needed since these reviews point towards substantial variability among study results, and identify sources of heterogeneity between studies in many study characteristics including stimulation parameters, target area, method of craving assessment, and clinical patient characteristics. So far, three meta-analyses have been conducted on this topic. The first included studies using either tDCS or TMS, and included studies focusing on craving for substances but also food [17]. In this meta-analysis, a medium effect size was reported (Hedge's $g=0.476$ ) in favor of active stimulation compared with sham stimulation in reducing craving. The other two, more recent meta-analyses narrowed down their inclusion criteria by including only randomized controlled trials using rTMS as the neuromodulation method. One found no overall difference between active and sham rTMS (Hedge's $g=0.043$ ), but further analyses based on type, site, and substance found that active stimulation was superior to sham stimulation in studies that stimulated the right DLPFC $[27 \cdot \bullet]$. The other meta-analysis found a medium effect size (Hedge's $g=0.75$ ) favoring active stimulation over placebo; when distinguishing nicotine use disorder and alcohol use disorder, the effect size was large for nicotine (Hedge's $g=$ 1.00 ) and no favorable effect was present for alcohol (Hedge's $g=-0.06$ ) [28]. The contradictory conclusions of these metaanalyses further highlight the variability in the effects of neuromodulation on craving in SUDs.

In gambling disorder, craving and its neural equivalent, cue reactivity, resemble the findings on the role of craving and cue reactivity in SUDs. Cue reactivity is the reactivity in the brain to addiction-relevant cues, compared with neutral, nonaddiction-related cues [29, 30]. For instance, increased neural cue reactivity in the striatum, putamen, orbitofrontal cortex, and insular cortex has been reported. I.e., when disordered gamblers are confronted with relevant gambling related cues, subcortical and fronto-striatal circuitry is increased in activity, and this is linked to higher self-reported craving [3, 31]. Only very recently, the first pilot studies on neuromodulation and its effects on craving in gambling disorder are emerging. Gay and colleagues [32••] studied 22 disordered gamblers who were in treatment, finding that a single-session high-frequency rTMS $(10 \mathrm{~Hz}, 94$ trains of $3.2 \mathrm{~s})$ over the left DLPFC reduced cueinduced craving more than placebo (sham) stimulation. This study had a cross-over design and used a commercial sham coil in combination with local electrical stimulation with electromyography electrodes using a transcutaneous electrical nerve stimulation (TENS) stimulator to optimize blinding. This advanced form of placebo stimulation prevents that 
participants in a cross-over study can discern active from sham stimulation by the differences in sensation on the skin. The findings for the active condition in this study thus cannot be attributed to placebo effects or unblinding; indeed, only $23 \%$ of participants guessed their stimulation allocation correctly. A further strength of this study was that neuronavigation was used to locate the DLPFC, thus optimizing the targeting of the stimulation site. A limitation of this study is the clinical validity of the outcome measure: the effects on cue-induced craving were measured directly after stimulation, but no changes in gambling behavior were found between active and sham rTMS in the seven days after stimulation.

In a study in 30 disordered gamblers, using a cross-over design with sham and active right DLPFC rTMS (1 Hz, $6 \mathrm{~min}$ ), reductions in craving were present in both the active and sham conditions [33••]. The large placebo effect in this study indicates that blinding in neuromodulation trials is of special importance. Such placebo effects have also been observed in pharmacological trials in gambling disorder, leading to speculation that gambling disorder is a condition that may be especially prone to placebo effects. This could be related to cognitive misperceptions present in gambling disorder, which for instance refer to thinking that one has control over random events present in gambling. As the allocation to placebo or active condition is also dependent on chance, specifically disordered gamblers may more frequently have a strong belief that they "are lucky," and are receiving the active medication or active neuromodulation. One other study investigated the effects of different forms of TMS on a construct related to craving, "desire to gamble," and on gambling behavior itself [34•]. In this study in nine disordered gamblers using a cross-over design, high-frequency rTMS $(10 \mathrm{~Hz}$, three times 15 trains of $1 \mathrm{~s})$ over right medial prefrontal cortex (mPFC) was compared with continuous theta burst TMS (cTBS: three times $50 \mathrm{~Hz}$ triplets repeated at $5 \mathrm{~Hz}, 20 \mathrm{~s}$ ) over right DLPFC as well as sham stimulation (vertex stimulation with an 8-shaped coil, perpendicular to the target area). The right DLPFC stimulation led to a decrease in desire to gamble scores after a session of slot machine play, whereas cTBS diminished diastolic blood pressure after slot machine play. We consider this study further in the sections on cognition and relapse.

\section{Neuromodulation and Effects on Cognition}

The dorsolateral prefrontal cortex (DLPFC) has a crucial role in higher cognitive functions like executive functions [35]. Executive functions have been shown to be impaired in SUDs [36], and further related to relapse in SUDs [9]. Enhancing DLPFC activity could result in increased cognitive functioning, which may be beneficial for treatment outcome in SUDs. Indeed, positive effects of non-invasive neuromodulation in SUD have been reported [37-49], as well as no effect [38, 41, 42, 44, 47, 50-54] and even in some rare cases negative effects [38, 43, 55].

Systematic reviews $[8 \bullet \bullet, 26 \bullet, 56]$ discussing these studies in more depth highlight mostly promising effects on executive or cognitive functioning, but also point to methodological variability between studies such as duration of sessions, number of sessions, target areas, and neuromodulation as add-on treatment or as a standalone intervention, all impairing comparability. In addition, most studies have small sample sizes, there is a lack of double-blind sham-controlled studies, and different neurocognitive tasks are implemented to measure constructs like decision-making and response inhibition. Even in instances when similar tasks are used, the outcome measures employed can differ between the studies. Differences in population characteristics such as treatment seeking status, duration of abstinence, and type and severity of substance use may influence the effect of neuromodulation on cognitive functions. To shed light on these questions, standardized neuromodulation protocols are recommended. In general, the field needs larger sham-controlled clinical trials in order to firmly establish the effects of neuromodulation on executive functions; however, most studies that are currently present do indicate a positive effect of neuromodulation on cognitive functions in SUDs.

As outlined in the introduction, disordered gambling has been associated with diminished cognitive-motivational functioning, as most consistently shown in executive functions and decision-making $[1 \bullet, 2-5]$. These functions in turn have been linked to relapse in SUDs [9], and in disordered gambling [10, 11]. Thus, improving cognitive-motivational functioning in disordered gambling may improve treatment effects. The number of studies investigating neuromodulation in disordered gambling is very limited as of yet, and for effects on cognitive functions, only three published studies are present: A study performed by Soyata and colleagues focused on the effects of tDCS on decision-making and flexibility in 20 disordered gamblers, using a cross-over design. Compared with sham, tDCS (anode right DLPFC, $2 \mathrm{~mA}, 35 \mathrm{~cm}^{2}, 20 \mathrm{~min}$ ) over the right DLPFC resulted in improvement in decision-making as measured with the Iowa gambling task, and in improvement of cognitive flexibility as measured with the Wisconsin Card Sorting Test [57••]. However, no long-term cognitive outcomes or clinical measures were included in this study, which can be viewed more as a neuroscientific study into working mechanisms of tDCS in gambling disorder. The study by Zack and colleagues, discussed in detail in the section on craving and neuromodulation above, indicated no changes of either $\mathrm{mPFC}$ high-frequency rTMS or cTBS on impulsive choice as measured with the delay discounting task, whereas interference effects on the Stroop became larger, contrary to expectations [34•]. Finally, Dickler et al. [58] describe the effect of one anodal tDCS session ( $\left.1 \mathrm{~mA}, 35 \mathrm{~mm}^{2}, 30 \mathrm{~min}\right)$ over the right DLPFC on gamma-aminobutyric acid (GABA), glutamate, and 
$\mathrm{N}$-acetyl aspartate (NAA) levels in the right DLPFC and right striatum. Active stimulation increased GABA concentrations in the right DLPFC; however, no significant changes in glutamate and NAA concentrations were observed. Also, no changes in metabolite concentrations were observed in the right striatum. Furthermore, correlations were performed between behavior (risk taking as assessed with the BART and impulsivity as assessed with the BIS-11) and metabolite levels during active stimulation. Positive correlations were found between risk taking and prefrontal glutamate, risk taking and striatal GABA, and impulsivity and striatal NAA. Authors suggest this implicates that when gambling disordered patients are more impulsive or more risk taking, they were more likely to respond to tDCS; however, no direct comparison was made for correlations between metabolite concentrations and sham stimulation.

\section{Effects of Non-invasive Neuromodulation on Relapse}

Substance use outcome measures have been reported in only a minority of neuromodulation trials in SUD populations [for reviews, see $\left.26^{\bullet}, 59^{*}\right]$. We will here elaborate on a few clinical trials with substantial (1-12 months) follow-up periods. For tDCS, one study applied five sessions of standalone tDCS treatment to participants that smoked at least ten cigarettes per day. Active stimulation significantly decreased cigarettes smoked per day and was modified by the level of motivation to quit smoking at baseline [60]. Of the four studies that applied tDCS in alcohol-dependent patients as add-on treatment with clinically relevant follow-up periods, two reported positive results on relapse $[54,61]$ and two reported no effect of tDCS compared with placebo on relapse [47, 52]. For an overview of these studies and their stimulation protocol, see Table 1. Altogether, based on the mixed results of the limited available studies using tDCS to reduce substance use, it is currently premature to draw firm conclusions on efficacy.

Next to tDCS, several clinical trials in SUDs with rTMS are present. Three larger rTMS studies in heavy smokers were conducted. The first showed significantly less relapse during treatment, although at follow-up, no significant differences between groups were found [62]. Another study found evidence for HF compared with LF and sham regarding nicotine intake, response rate, and reduction in cigarettes consumed at six months follow-up [63]. The third study did not find differences in cigarette consumption at six-month follow-up [64]. Regarding alcohol as substance of use, two clinical trials are available. The first study showed a positive effect on several outcomes related to alcohol use or relapse during the fourweek treatment, but no longer follow-up period was conducted in this study [65]. The other study found decreased number of drinks consumed daily up to three months in the active group, while this pattern was not found in the sham group 
(note however that no comparison between the active and sham group was conducted) [66]. Besides nicotine and alcohol, two pilot studies in cocaine use disorder are present. In the first study, outpatient cocaine-dependent individuals showed decreased cocaine intake in the active group and not in the placebo group (note: direct comparison between groups was not significant) [67]. In the second, open-label pilot study, decreased cocaine use after rTMS treatment compared with medical treatment only was found [68]. For an overview of these studies and their stimulation protocol, see Table 2. Summarizing these results, the effect of neuromodulation on substance use is scarcely studied and results are not at all conclusive. Therefore, further studies are needed before any conclusions can be drawn. The field is in need of studies that are sham controlled; at least single blind and conducted in larger clinical samples where tDCS or rTMS is added to conventional evidence-based therapies for SUD, as indicated in reviews $[8 \cdot \bullet, 26 \bullet, 59 \bullet \cdot$. As reducing or abstaining from substances is the main goal of SUD treatment, it is highly relevant for future studies to include clinically relevant follow-up periods assessing substance use.

Of the three studies in gambling disorder that used neuromodulation and studied effects on actual gambling behavior or clinical gambling scales, two studies are single-session studies: one assessed acute gambling behavior (on a lab-based slot machine) directly after single-session rTMS, cTBS, or sham stimulation [34•], and no effects on acute gambling behavior were present. In another study, no effects of a single session of left DLPFC stimulation vs sham stimulation on gambling behavior in the week after stimulation were present [32••]. Finally, in a case series study in five disordered gamblers, effects of 15 daily sessions with deep rTMS, using an $\mathrm{H} 1$ coil, with a 1-Hz inhibitory DLPFC protocol were investigated. This case series in four participants (the fifth patient dropped out) did result in improvement on clinical scales, ranging from the Hamilton Depression Rating Scale to the Yale-Brown Obsessive Compulsive Scale, a non-specified visual analogue scale, and the South Oaks Gambling Screen after the last rTMS session [69]. Although the authors report diminished scores after 15 sessions (in three patients) and after two session (one patient), information from colaterals indicated no improvement in problem gambling. This led the authors to conclude that the $1-\mathrm{Hz}$ stimulation was not effective, and that an excitatory stimulation (e.g., $10 \mathrm{~Hz}$ ) may have differential effects. See Table 3 for an overview of the studies conducted in gambling disorder.

Clearly, from the first two studies, it is evident that these studies were not clinical trials, designed to investigate longterm effects of neuromodulation on diminishing problematic gambling. The third case series study actually was the first one to employ TMS in gambling disorder, but only concerns four disordered gamblers. Thus, the first clinical trial studies are still needed addressing the clinical potential of neuromo dulation in gambling disorder. 


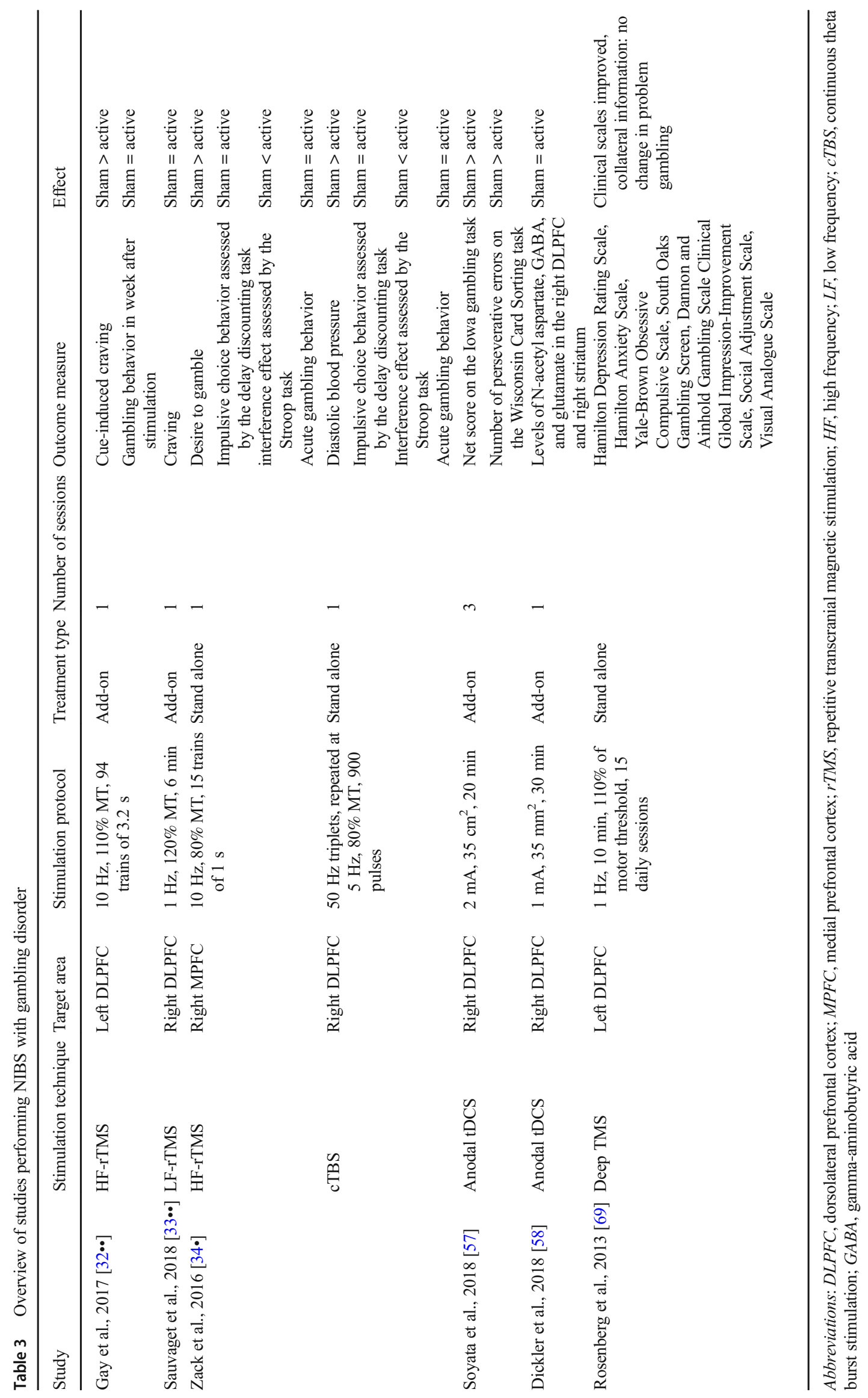




\section{Conclusion and Discussion}

In reviewing the current evidence for neuromodulation as a treatment target in addiction, and its specific implications for gambling disorder, it can be concluded that neuromodulation targets relevant working mechanisms related to development, course, and relapse in SUDs. Several reviews and metaanalyses indicate that neuromodulation in SUDs has a beneficial effect on craving and cognitive functions $[8 \cdot \bullet, 18]$. At the same time, the evidence for effects of neuromodulation on clinical outcome measures in addiction is still limited. For gambling disorder, a mere six studies on neuromodulation are present that investigated outcome measures ranging from gambling urges, craving, cognitive flexibility, and decision-making to gambling behavior directly following neuromodulation. Clearly, the field is in need of larger studies.

The current studies in gambling disorder all employed singlesession (cross-over) designs and thus, the field is in need of studies that also focus on multiple-session neuromodulation protocols, as the potential to have longer-term effects on craving, cognition, and clinical outcome measures is higher for multiplesession neuromodulation trials. In this respect, clinical trials in depression could be used as a starting point, because rTMS is now approved in several countries as a treatment method for treatment-refractory depression. In gambling disorder, several evidence-based treatment strategies are present, with larger effect sizes for psychosocial interventions compared with pharmacological interventions [70, 71]. It is possible that the add-on of neuromodulation to psychosocial treatment methods, like cognitive behavioral therapy or motivational interviewing, may render the brain more flexible, thus enhancing treatment effects. An alternative working mechanism may be that DLPFC stimulation by rTMS or neuromodulation by tDCS may enhance cognitive control over craving, by improving DLPFC functioning. With regular rTMS targeted at the DLPFC, changes in striatal dopamine binding in depressed patients indicate that multiple sessions of high-frequency rTMS can induce an increase in striatal dopamine release [72]. Newer technological advances in neuromodulation may broaden possibilities for neuromodulation in addictive disorders as well. For example, deep rTMS has been shown to enable subcortical changes in dopamine functioning, by changing dopamine transporter availability in alcoholdependent patients [65], and this may render larger clinical effects.

Besides the need for multiple-session rTMS studies, sample sizes need to be increased in order for the field to move beyond pilot studies, as currently, the studies are very small. In addition, rTMS seems to be associated with a high placebo response, which exists in pharmacological studies in disordered gambling as well [71]. Well-controlled trials employing sham stimulation protocols, including formal assessment of blinding in participants, are needed to overcome this problem. Cross-over designs may not be ideal for blinding, although recent studies employing commercially available sham coils in combination with local electrical stimulation with electromyography electrodes can optimize blinding [32••].

As current therapies for disordered gambling have a comparable treatment efficacy with those of SUDs and other psychiatric disorders, there is a clear possibility for the improvement of treatment effects. While neuromodulation still has a long way to go in terms of clinical evidence base in SUDs and gambling disorder, the available treatment options for addictive disorders are all cost-effective. Therefore, there is no reason why costeffectiveness would not be possible for neuromodulation, as the availability of neuromodulation equipment, like more costly TMS machines, will increase, now that it is approved for other psychiatric disorders, like treatment-refractory depression and obsessive compulsive disorder recently.

In conclusion, although the number of studies employing neuromodulation in gambling disorder is limited, and there is no evidence yet from formal RCTs in gambling disorder, there are indications that neuromodulation can diminish craving and improve cognitive functions in gambling disorder. As evidence from SUDs regarding the effects of neuromodulation on craving and cognitive functions is promising, disordered gambling may benefit from neuromodulation, not only through a direct effect on reducing gambling problems but also through enhancing executive functions, thus improving control over craving, or through diminishing craving, potentially through subcortical changes induced by neuromodulation.

\section{Compliance with Ethical Standards}

Conflict of Interest Dr. Goudriaan reports grants from ZonMw, Health Research The Netherlands, outside the submitted work. R. S. Schluter has nothing to disclose.

Human and Animal Rights and Informed Consent This article does not contain any studies with human or animal subjects performed by any of the authors.

Open Access This article is distributed under the terms of the Creative Commons Attribution 4.0 International License (http:// creativecommons.org/licenses/by/4.0/), which permits unrestricted use, distribution, and reproduction in any medium, provided you give appropriate credit to the original author(s) and the source, provide a link to the Creative Commons license, and indicate if changes were made.

\section{References}

Papers of particular interest, published recently, have been highlighted as:

- Of importance

•- Of major importance

1. van Timmeren T, Daams JG, van Holst RJ, Goudriaan AE. Compulsivity-related neurocognitive performance deficits in 
gambling disorder: a systematic review and meta-analysis. Neurosci Biobehav Rev. 2018;84:204-17. https://doi.org/10. 1016/j.neubiorev.2017.11.022. This systematic review indicates that cognitive flexibility and other compulsivity-related neurocognitive performance deficits in gambling disorders are present, functions which are malleable by neuromodulation, as evidenced in substance use disorders, and therefore also relevant for gambling disorder neuromodulation studies.

2. Kertzman S, Lowengrub K, Aizer A, Vainder M, Kotler M, Dannon PN. Go-no-go performance in pathological gamblers. Psychiatry Res. 2008;161(1):1-10. https://doi.org/10.1016/j.psychres.2007. 06.026 .

3. van Holst RJ, van den Brink W, Veltman DJ, Goudriaan AE. Why gamblers fail to win: a review of cognitive and neuroimaging findings in pathological gambling. Neurosci Biobehav Rev. 2010;34(1):87-107. https://doi.org/10.1016/j.neubiorev.2009.07. 007.

4. Michalczuk R, Bowden-Jones H, Verdejo-Garcia A, Clark L. Impulsivity and cognitive distortions in pathological gamblers attending the UK National Problem Gambling Clinic: a preliminary report. Psychol Med. 2011;41:1-11. https://doi.org/10.1017/ S003329171100095X.

5. Goudriaan AE, Oosterlaan J, de Beurs E, van den Brink W. Decision making in pathological gambling: a comparison between pathological gamblers, alcohol dependents, persons with Tourette syndrome, and normal controls. Brain Res Cogn Brain Res. 2005;23(1):137-51. https://doi.org/10.1016/j.cogbrainres.2005.01. 017.

6. Lannoy S, Maurage P, D’Hondt F, Billieux J, Dormal V. Executive impairments in binge drinking: evidence for a specific performance-monitoring difficulty during alcohol-related processing. Eur Addict Res. 2018;24(3):118-27. https://doi.org/10.1159/ 000490492.

7. Briere M, Tocanier L, Allain P, Le Gal D, Allet G, Gorwood P, et al. Decision-making measured by the Iowa gambling task in patients with alcohol use disorders choosing harm reduction versus relapse prevention program. Eur Addict Res. 2019;25:1-9. https://doi.org/ $10.1159 / 000499709$.

8.• Schluter RS, Daams JG, van Holst RJ, Goudriaan AE. Effects of non-invasive neuromodulation on executive and other cognitive functions in addictive disorders: a systematic review. Front Neurosci. 2018;12:642. https://doi.org/10.3389/fnins.2018.00642 In this systematic review (PRISMA), effects of neuromodulation on executive and related cognitive functions in substance use disorders are reviewed. In the majority of studies, either improvement or no effects are reported, whereas negative effects of neuromodulation are only present in a few studies. As gambling disorder also is associated with diminished executive functions, this review points to relevant potential working mechanisms of neuromodulation for gambling disorder.

9. Stevens L, Verdejo-Garcia A, Goudriaan AE, Roeyers H, Dom G, Vanderplasschen W. Impulsivity as a vulnerability factor for poor addiction treatment outcomes: a review of neurocognitive findings among individuals with substance use disorders. J Subst Abus Treat. 2014;47(1):58-72. https://doi.org/10.1016/j.jsat.2014.01. 008.

10. Goudriaan AE, Oosterlaan J, de Beurs E, van den Brink W. The role of self-reported impulsivity and reward sensitivity versus neurocognitive measures of disinhibition and decision-making in the prediction of relapse in pathological gamblers. Psychol Med. 2008;38(1):41-50.

11. Alvarez-Moya EM, Ochoa C, Jimenez-Murcia S, Aymami MN, Gomez-Pena M, Fernandez-Aranda F, et al. Effect of executive functioning, decision-making and self-reported impulsivity on the treatment outcome of pathologic gambling. J Psychiatry Neurosci. 2011;36(3):165-75. https://doi.org/10.1503/jpn.090095.

12. Huerta PT, Volpe BT. Transcranial magnetic stimulation, synaptic plasticity and network oscillations. J Neuroeng Rehabil. 2009;6:7. https://doi.org/10.1186/1743-0003-6-7.

13. Perera T, George MS, Grammer G, Janicak PG, Pascual-Leone A, Wirecki TS. The clinical TMS society consensus review and treatment recommendations for TMS therapy for major depressive disorder. Brain Stimul. 2016;9(3):336-46. https://doi.org/10.1016/j. brs.2016.03.010.

14. FDA. FDA permits marketing of transcranial magnetic stimulation for treatment of obsessive compulsive disorder. 2018. https://www. fda.gov/NewsEvents/Newsroom/PressAnnouncements/ ucm617244.htm. Accessed 03-06-2019 2019.

15. Oberman L, Edwards D, Eldaief M, Pascual-Leone A. Safety of theta burst transcranial magnetic stimulation: a systematic review of the literature. J Clin Neurophysiol. 2011;28(1):67-74. https://doi. org/10.1097/WNP.0b013e318205135f.

16. Nitsche MA, Paulus W. Excitability changes induced in the human motor cortex by weak transcranial direct current stimulation. J Physiol. 2000;527(Pt 3):633-9. https://doi.org/10.1111/j.14697793.2000.t01-1-00633.x.

17. Jansen JM, Daams J, Koeter MW, Veltman DJ, van den Brink W, Goudriaan AE. Effects of non-invasive neuro-stimulation on craving: a meta-analysis. Neurosci Biobehav Rev. 2013;37(10):247280 .

18. Trojak B, Sauvaget A, Fecteau S, Lalanne L, Chauvet-Gelinier JC, Koch S, et al. Outcome of non-invasive brain stimulation in substance use disorders: a review of randomized sham-controlled clinical trials. J Neuropsychiatry Clin Neurosci. 2017;29(2):105-18. https://doi.org/10.1176/appi.neuropsych.16080147.

19. Bellamoli E, Manganotti P, Schwartz RP, Rimondo C, Gomma M, Serpelloni G. rTMS in the treatment of drug addiction: an update about human studies. Behav Neurol. 2014;2014:815215-1. https:// doi.org/10.1155/2014/815215.

20. Bolloni C, Badas P, Corona G, Diana M. Transcranial magnetic stimulation for the treatment of cocaine addiction: evidence to date. Subst Abus Rehabil. 2018;9:11-21. https://doi.org/10.2147/SAR. S161206.

21. Gorelick DA, Zangen A, George MS. Transcranial magnetic stimulation in the treatment of substance addiction. Ann N Y Acad Sci. 2014;1327:79-93. https://doi.org/10.1111/nyas.12479.

22. Herremans SC, Baeken C. The current perspective of neuromodulation techniques in the treatment of alcohol addiction: a systematic review. Psychiatr Danub. 2012;24(Suppl 1):S14-20.

23. Hone-Blanchet A, Ciraulo DA, Pascual-Leone A, Fecteau S. Noninvasive brain stimulation to suppress craving in substance use disorders: review of human evidence and methodological considerations for future work. Neurosci Biobehav Rev. 2015;59:184 200. https://doi.org/10.1016/j.neubiorev.2015.10.001.

24. Lapenta OM, Marques LM, Rego GG, Comfort WE, Boggio PS. tDCS in addiction and impulse control disorders. J ECT. 2018;34(3):182-92. https://doi.org/10.1097/YCT. 0000000000000541 .

25. Salling MC, Martinez D. Brain stimulation in addiction. Neuropsychopharmacology. 2016;41(12):2798-809. https://doi. org/10.1038/npp.2016.80.

26. Stein ER, Gibson BC, Votaw V, Wilson AD, Clark VP, Witkiewitz $\mathrm{K}$. Non-invasive brain stimulation in substance use disorders: implications for dissemination to clinical settings. Curr Opin Psychol. 2018. https://doi.org/10.1016/j.copsyc.2018.12.009 Although this review is not a systematic review, it discusses neurostimulation studies in substance use disorders with all relevant outcome measures: craving, cognition, and substance use or relapse, and thus provides a comprehensive review. 
27.• Enokibara M, Trevizol A, Shiozawa P, Cordeiro Q. Establishing an effective TMS protocol for craving in substance addiction: is it possible? Am J Addict. 2016;25(1):28-30. https://doi.org/10. 1111/ajad.12309 This recent meta-analysis indicates that the effects of TMS on craving in substance use disorders may differ between substances, which is important for future studies in SUDs. As this article is a meta-analysis, it provides a review including the effect sizes and comparison between studies regarding their effects.

28. Maiti R, Mishra BR, Hota D. Effect of high-frequency transcranial magnetic stimulation on craving in substance use disorder: a metaanalysis. J Neuropsychiatry Clin Neurosci. 2017;29(2):160-71. https://doi.org/10.1176/appi.neuropsych.16040065.

29. Goudriaan AE, de Ruiter MB, van den Brink W, Oosterlaan J, Veltman DJ. Brain activation patterns associated with cue reactivity and craving in abstinent problem gamblers, heavy smokers and healthy controls: an fMRI study. Addict Biol. 2010;15(4):491503. https://doi.org/10.1111/j.1369-1600.2010.00242.x.

30. van Holst RJ, van Holstein M, van den Brink W, Veltman DJ, Goudriaan AE. Response inhibition during cue reactivity in problem gamblers: an fMRI study. PLoS One. 2012;7(3):e30909. https://doi.org/10.1371/journal.pone.0030909.

31. Goudriaan AE, Yucel M, van Holst RJ. Getting a grip on problem gambling: what can neuroscience tell us? Front Behav Neurosci. 2014;8:141. https://doi.org/10.3389/fnbeh.2014.00141.

32.• Gay A, Boutet C, Sigaud T, Kamgoue A, Sevos J, Brunelin J, et al. A single session of repetitive transcranial magnetic stimulation of the prefrontal cortex reduces cue-induced craving in patients with gambling disorder. Eur Psychiatry. 2017;41:68-74. https://doi.org/ 10.1016/j.eurpsy.2016.11.001 One of the few neuromodulation studies in gambling disorder. Results indicate that a single session of high-frequency rTMS reduces cue-induced craving in gambling disorder, but has no effects on gambling behavior in the seven days after the single-session stimulation.

33.• Sauvaget A, Bulteau S, Guilleux A, Leboucher J, Pichot A, Valriviere $\mathrm{P}$, et al. Both active and sham low-frequency rTMS single sessions over the right DLPFC decrease cue-induced cravings among pathological gamblers seeking treatment: a randomized, double-blind, sham-controlled crossover trial. J Behav Addict. 2018;7(1):126-36. https://doi.org/10.1556/2006.7.2018.14 One of the few neurostimulation studies in gambling disorder. Results indicate that both sham-stimulation and highfrequency rTMS stimulation result in diminished craving, pointing to a high placebo response in gambling disorder. This has high relevance, as blinding deserves attention in future neuromodulation studies in gambling disorder.

34. Zack M, Cho SS, Parlee J, Jacobs M, Li C, Boileau I, et al. Effects of high frequency repeated transcranial magnetic stimulation and continuous theta burst stimulation on gambling reinforcement, delay discounting, and Stroop interference in men with pathological gambling. Brain Stimul. 2016;9(6):867-75. https://doi.org/10. 1016/j.brs.2016.06.003 One of the few neurostimulation studies in gambling disorder, in a small sample of nine male disordered gamblers. Both high-frequency rTMS, continuous theta burst stimulation (cTBS) and a sham stimulation were performed in a cross-over design. Mixed effects were found on a range of outcome measures: high-frequency rTMS resulted in a decrease in gambling urge, whereas cTBS resulted in diminished blood pressure. No effects of the active vs sham stimulation were present on delay discounting, a measure of impulsive choice, and interference scores on the Stroop, a cognitive interference measure, even increased.

35. Ridderinkhof KR, van den Wildenberg WP, Segalowitz SJ, Carter CS. Neurocognitive mechanisms of cognitive control: the role of prefrontal cortex in action selection, response inhibition, performance monitoring, and reward-based learning. Brain Cogn. 2004;56(2):129-40. https://doi.org/10.1016/j.bandc.2004.09.016.

36. Verdejo-Garcia A, Bechara A, Recknor EC, Perez-Garcia M. Executive dysfunction in substance dependent individuals during drug use and abstinence: an examination of the behavioral, cognitive and emotional correlates of addiction. J Int Neuropsychol Soc. 2006;12(3):405-15.

37. Del Felice A, Bellamoli E, Formaggio E, Manganotti P, Masiero S, Cuoghi G, et al. Neurophysiological, psychological and behavioural correlates of rTMS treatment in alcohol dependence. Drug Alcohol Depend. 2016;158:147-53. https://doi.org/10.1016/j. drugalcdep.2015.11.018.

38. Sheffer CE, Mennemeier M, Landes RD, Bickel WK, Brackman S, Dornhoffer J, et al. Neuromodulation of delay discounting, the reflection effect, and cigarette consumption. J Subst Abus Treat. 2013;45(2):206-14. https://doi.org/10.1016/j.jsat.2013.01.012.

39. Herremans SC, Vanderhasselt MA, De Raedt R, Baeken C. Reduced intra-individual reaction time variability during a GoNoGo task in detoxified alcohol-dependent patients after one right-sided dorsolateral prefrontal HF-rTMS session. Alcohol Alcohol. 2013;48(5):552-7. https://doi.org/10.1093/alcalc/agt054.

40. Qiao J, Jin G, Lei L, Wang L, Du Y, Wang X. The positive effects of high-frequency right dorsolateral prefrontal cortex repetitive transcranial magnetic stimulation on memory, correlated with increases in brain metabolites detected by proton magnetic resonance spectroscopy in recently detoxified alcohol-dependent patients. Neuropsychiatr Dis Treat. 2016;12:2273-8. https://doi.org/10. 2147/NDT.S106266.

41. Su H, Zhong N, Gan H, Wang J, Han H, Chen T, et al. High frequency repetitive transcranial magnetic stimulation of the left dorsolateral prefrontal cortex for methamphetamine use disorders: a randomised clinical trial. Drug Alcohol Depend. 2017;175:84-91. https://doi.org/10.1016/j.drugalcdep.2017.01.037.

42. Fecteau S, Agosta S, Hone-Blanchet A, Fregni F, Boggio P, Ciraulo $\mathrm{D}$, et al. Modulation of smoking and decision-making behaviors with transcranial direct current stimulation in tobacco smokers: a preliminary study. Drug Alcohol Depend. 2014;140:78-84. https:// doi.org/10.1016/j.drugalcdep.2014.03.036.

43. Gorini A, Lucchiari C, Russell-Edu W, Pravettoni G. Modulation of risky choices in recently abstinent dependent cocaine users: a transcranial direct-current stimulation study. Front Hum Neurosci. 2014;8:661. https://doi.org/10.3389/fnhum.2014.00661.

44. Pripfl J, Neumann R, Kohler U, Lamm C. Effects of transcranial direct current stimulation on risky decision making are mediated by 'hot' and 'cold' decisions, personality, and hemisphere. Eur J Neurosci. 2013;38(12):3778-85. https://doi.org/10.1111/ejn. 12375 .

45. den Uyl TE, Gladwin TE, Wiers RW. Transcranial direct current stimulation, implicit alcohol associations and craving. Biol Psychol. 2015;105:37-42. https://doi.org/10.1016/j.biopsycho.2014.12.004.

46. Hoppner J, Broese T, Wendler L, Berger C, Thome J. Repetitive transcranial magnetic stimulation (rTMS) for treatment of alcohol dependence. World J Biol Psychiatry. 2011;12(Suppl 1):57-62. https://doi.org/10.3109/15622975.2011.598383.

47. da Silva MC, Conti CL, Klauss J, Alves LG, do Nascimento Cavalcante HM, Fregni F, et al. Behavioral effects of transcranial direct current stimulation (tDCS) induced dorsolateral prefrontal cortex plasticity in alcohol dependence. J Physiol Paris. 2013;107(6):493-502. https://doi.org/10.1016/j.jphysparis. 2013. 07.003 .

48. Smith RC, Boules S, Mattiuz S, Youssef M, Tobe RH, Sershen H, et al. Effects of transcranial direct current stimulation (tDCS) on cognition, symptoms, and smoking in schizophrenia: a randomized controlled study. Schizophr Res. 2015;168(1-2):260-6. https://doi. org/10.1016/j.schres.2015.06.011. 
49. Nakamura-Palacios EM, de Almeida Benevides MC, da Penha ZGM, de Oliveira RW, de Vasconcellos VF, de Castro LN, et al. Auditory event-related potentials (P3) and cognitive changes induced by frontal direct current stimulation in alcoholics according to Lesch alcoholism typology. Int J Neuropsychopharmacol. 2012;15(5):601-16. https://doi.org/10.1017/S1461145711001040.

50. Xu J, Fregni F, Brody AL, Rahman AS. Transcranial direct current stimulation reduces negative affect but not cigarette craving in overnight abstinent smokers. Front Psychiatry. 2013;4:112. https://doi. org/10.3389/fpsyt.2013.00112.

51. Huang W, Shen F, Zhang J, Xing B. Effect of repetitive transcranial magnetic stimulation on cigarette smoking in patients with schizophrenia. Shanghai Arch Psychiatry. 2016;28(6):309-17. https://doi. org/10.11919/j.issn.1002-0829.216044.

52. den Uyl TE, Gladwin TE, Rinck M, Lindenmeyer J, Wiers RW. A clinical trial with combined transcranial direct current stimulation and alcohol approach bias retraining. Addict Biol. 2017;22(6): 1632-40. https://doi.org/10.1111/adb.12463.

53. den Uyl TE, Gladwin TE, Wiers RW. Electrophysiological and behavioral effects of combined transcranial direct current stimulation and alcohol approach bias retraining in hazardous drinkers. Alcohol Clin Exp Res. 2016;40(10):2124-33. https://doi.org/10. 1111/acer.13171.

54. Klauss J, Penido Pinheiro LC, Silva Merlo BL, de Almeida Correia Santos G, Fregni F, Nitsche MA, et al. A randomized controlled trial of targeted prefrontal cortex modulation with tDCS in patients with alcohol dependence. Int J Neuropsychopharmacol. 2014;17(11):1793-803. https://doi.org/10.1017/ S1461145714000984.

55. Boggio PS, Zaghi S, Villani AB, Fecteau S, Pascual-Leone A, Fregni F. Modulation of risk-taking in marijuana users by transcranial direct current stimulation (tDCS) of the dorsolateral prefrontal cortex (DLPFC). Drug Alcohol Depend. 2010;112(3):220-5. https://doi.org/10.1016/j.drugalcdep.2010.06.019.

56. Naish KR, Vedelago L, MacKillop J, Amlung M. Effects of neuromodulation on cognitive performance in individuals exhibiting addictive behaviors: a systematic review. Drug Alcohol Depend. 2018;192:338-51. https://doi.org/10.1016/j.drugalcdep. 2018.08.018.

57.• Soyata AZ, Aksu S, Woods AJ, Iscen P, Sacar KT, Karamursel S. Effect of transcranial direct current stimulation on decision making and cognitive flexibility in gambling disorder. Eur Arch Psychiatry Clin Neurosci. 2018. https://doi.org/10.1007/s00406-018-0948-5 This is one of the few neuromodulation studies in gambling disorder. In this tDCS cross-over study in twenty disordered gamblers, anodal right DLPFC modulation resulted in improvement in decision-making as measured with the Iowa gambling task, and in improvement of cognitive flexibility as measured with the Wisconsin Card Sorting Test.

58. Dickler M, Lenglos C, Renauld E, Ferland F, Edden RA, Leblond J, et al. Online effects of transcranial direct current stimulation on prefrontal metabolites in gambling disorder. Neuropharmacology. 2018;131:51-7. https://doi.org/10.1016/j.neuropharm.2017.12. 002.

59.• Coles AS, Kozak K, George TP. A review of brain stimulation methods to treat substance use disorders. Am J Addict. 2018;27(2):71-91. https://doi.org/10.1111/ajad.12674 This is the first systematic review (non-PRISMA) on the effect of neuromodulation on relapse in substance use disorders, thus discussing the evidence regarding neuromodulation on a clinically relevant outcome measure.

60. Brangioni MCVD, Pereira DA, Thibaut A, Fregni F, Brasil-Neto JP, Boechat-Barros R. Effects of prefrontal transcranial direct current stimulation and motivation to quit in tobacco smokers: a randomized, sham controlled, double-blind trial. Front Pharmacol. 2018;9. https://doi.org/10.3389/fphar.2018.00014.

61. Klauss J, Anders QS, Felippe LV, Nitsche MA, Nakamura-Palacios EM. Multiple sessions of transcranial direct current stimulation (tDCS) reduced craving and relapses for alcohol use: a randomized placebo-controlled trial in alcohol use disorder. Front Pharmacol. 2018;9:716. https://doi.org/10.3389/fphar.2018.00716.

62. Trojak B, Meille V, Achab S, Lalanne L, Poquet H, Ponavoy E, et al. Transcranial magnetic stimulation combined with nicotine replacement therapy for smoking cessation: a randomized controlled trial. Brain Stimul. 2015;8(6):1168-74. https://doi.org/10. 1016/j.brs.2015.06.004.

63. Dinur-Klein L, Dannon P, Hadar A, Rosenberg O, Roth Y, Kotler $\mathrm{M}$, et al. Smoking cessation induced by deep repetitive transcranial magnetic stimulation of the prefrontal and insular cortices: a prospective, randomized controlled trial. Biol Psychiatry. 2014;76(9): 742-9. https://doi.org/10.1016/j.biopsych.2014.05.020.

64. Amiaz R, Levy D, Vainiger D, Grunhaus L, Zangen A. Repeated high-frequency transcranial magnetic stimulation over the dorsolateral prefrontal cortex reduces cigarette craving and consumption. Addiction. 2009;104(4):653-60.

65. Addolorato G, Antonelli M, Cocciolillo F, Vassallo GA, Tarli C, Sestito L, et al. Deep transcranial magnetic stimulation of the dorsolateral prefrontal cortex in alcohol use disorder patients: effects on dopamine transporter availability and alcohol intake. Eur Neuropsychopharmacol. 2017;27(5):450-61. https://doi.org/10. 1016/j.euroneuro.2017.03.008.

66. Ceccanti M, Inghilleri M, Attilia ML, Raccah R, Fiore M, Zangen A, et al. Deep TMS on alcoholics: effects on cortisolemia and dopamine pathway modulation. A pilot study. Can J Physiol Pharmacol. 2015;93(4):283-90. https://doi.org/10.1139/cjpp2014-0188.

67. Bolloni C, Panella R, Pedetti M, Frascella AG, Gambelunghe C, Piccoli T, et al. Bilateral transcranial magnetic stimulation of the prefrontal cortex reduces cocaine intake: a pilot study. Front Psychiatry. 2016;7:133. https://doi.org/10.3389/fpsyt.2016.00133.

68. Terraneo A, Leggio L, Saladini M, Ermani M, Bonci A, Gallimberti L. Transcranial magnetic stimulation of dorsolateral prefrontal cortex reduces cocaine use: a pilot study. Eur Neuropsychopharmacol. 2016;26(1):37-44. https://doi.org/10.1016/j.euroneuro.2015.11. 011.

69. Rosenberg O, Klein LD, Dannon PN. Deep transcranial magnetic stimulation for the treatment of pathological gambling. Psychiatry Res. 2013;206(1):111-3. https://doi.org/10.1016/j.psychres.2012. 09.045 .

70. Pallesen S, Mitsem M, Kvale G, Johnsen BH, Molde H. Outcome of psychological treatments of pathological gambling: a review and meta-analysis. Addiction. 2005;100(10):1412-22. https://doi.org/ 10.1111/j.1360-0443.2005.01204.x.

71. Pallesen S, Molde H, Arnestad HM, Laberg JC, Skutle A, Iversen E, et al. Outcome of pharmacological treatments of pathological gambling: a review and meta-analysis. J Clin Psychopharmacol. 2007;27(4):357-64. https://doi.org/10.1097/jcp.013e3180dcc304d.

72. Pogarell O, Koch W, Popperl G, Tatsch K, Jakob F, Zwanzger P, et al. Striatal dopamine release after prefrontal repetitive transcranial magnetic stimulation in major depression: preliminary results of a dynamic [123I] IBZM SPECT study. J Psychiatr Res. 2006;40(4): 307-14. https://doi.org/10.1016/j.jpsychires.2005.09.001.

Publisher's Note Springer Nature remains neutral with regard to jurisdictional claims in published maps and institutional affiliations. 